\title{
Application of $\mathrm{THz}$ radiation for in situ control of eye cornea hydration level
}

\author{
$\underline{\text { I.Ozheredov }}^{1}$, M.Prokopchuk ${ }^{1}$, T.Safonova ${ }^{2}$, E.Sikach ${ }^{2}$, P.Solyankin ${ }^{3}$, A.Angeluts ${ }^{1}$, \\ A.Balakin ${ }^{1,3}$ and A.Shkurinov ${ }^{1,3}$ \\ ${ }^{1}$ Lomonosov Moscow State University, Moscow, Russian Federation, ozheredov@physics.msu.ru \\ ${ }^{2}$ Eye Diseases Institute, Moscow, Russian Federation \\ ${ }^{3}$ Institute on Laser and Information Technologies of RAS, Branch of the FSRC \\ "Crystallography and Photonics" RAS, Shatura, Moscow Region, Russian Federation
}

The epithelium of the cornea and conjunctiva needs continuous moistening. With closed eyelids, the tear fluid fills the entire space, and when open, it spreads over the anterior surface of the eye forming a thin film. Tear film has a thickness of $4-7 \mu \mathrm{m}$ and performs protective, metabolic and optical functions. The direct involvement of the corneal and conjunctival epithelium into formation of tear film was demonstrated in [1]. The tear film covers the whole corneal and conjunctival surface, from the area of transition from the keratinous multilayered epithelium of the eyelid skin edges to the non-corroborating multilayer epithelium of the conjunctiva and up to the corneal center. Thus it allows considering the tear film as one of the corneal layers.

Numerous causes leading to the loss of normal properties and structure of the tear film, lead to changes in the water-electrolyte balance. They also cause a decrease in the volume of tear fluid, a decrease in sensitivity and a development of corneal epitheliopathy. A complex of signs of the corneal and conjunctival epithelium lesion due to a decrease in the quality and/or amount of tear fluid defines a concept of 'dry eye syndrome' [2].

The assessment of pre-corneal tear film is a leading direction in the diagnosis of dry eye syndrome. In clinical practice the most common method for determining the stability of the tear film is Norn test. Non-invasive methods for studying the stability of a tear film include thioscopy. Confocal microscopy expands the possibilities of studying the anatomy of the cornea at the level of its microstructure. It has been shown recently $[3,4]$ for in vivo measurements of corneal tissue hydration and tear film dynamic control the terahertz $(\mathrm{THz})$ reflectometry might be efficiently used.

In the present study we apply frequencydomain $\mathrm{THz}$ reflectometry technique for sensing of the ocular surface system. Our study allows the dynamic control of pre-corneal tear film thinning and sensing of corneal tissue hydration level in clinical applications.

The concept of the proposed technique was based on $\mathrm{THz}$ photomixing approach. Typical photomixer consists of two independent tunable laser sources yielding the difference frequency in a desired $\mathrm{THz}$ region by heterodyning. Two laser beams with different frequencies light a photoconductive antenna (PCA), where running fringes with the $\mathrm{THz}$ difference frequency excite carries in the semiconductor material. The solid-state lasers are used as elements of a laser heterodyne. The single-mode selection of the laser is done using distributed feedback (DFB) - the diffraction grating technologically etched close to the p-n junction of the diode structure. PCA consists of an electrical dipole and semiconductor quick enough to produce carriers in time with the beat frequency. When the heterodyned laser beams light the surface of the semiconductor, than carriers appear in the material. If the metal antenna is polarized with some voltage then carriers give a periodical short circuit for the antenna and PCA converts the photocurrent into a $\mathrm{THz}$ wave.

The proposed apparatus was based on two DFB diode lasers with precise resonator temperature control. The lasers generated narrow $10 \mathrm{kHz}$ line in the range of $1530-1608 \mathrm{~nm}$ with average power of $22 \mathrm{~mW}$ each. X-type fiber optical beam splitter with the splitting ratio 50/50 combined both lasers output. The outputs of the splitter were connected to the $\mathrm{THz}$ emitter and receiver - low-temperature InGaAs bowtie PCA (BATOP GmbH). The bias voltage for the $\mathrm{THz}$ emitter $(0 / 6 \mathrm{~V}, 40 \mathrm{kHz})$ was provided by the function generator. The difference frequency range of DFB lasers allowed tuning of the output $\mathrm{THz}$ radiation within a range of $0.04-0.1 \mathrm{THz}$. High-density polyethylene lens (HDPE) with focal distance of $30 \mathrm{~mm}$ focused $\mathrm{THz}$ signal to the ocular surface system. The specularly reflected $\mathrm{THz}$ signal was guided through second similar HDPE-lens into the receiver PCA module. The digital lock-in amplifier SR 810 (Stanford Research Systems) was used to measure the resulting photocurrent. Both $\mathrm{THz}$ emitter and receiver were were embeded into the clinical ophthalmic apparatus (Fig.1) allowed measuring the eye reflection in $\mathrm{THz}$ range simultaneously with using of the other standard ophthalmological methodic.

For the in vivo study the special experimental protocol was developed. For each human eye cornea the reflected signal versus time was measured and reflection coefficient was calculated. Each measurement started at the time of eye opening. During the measurement series the testee human was requested to keep the eye open as long as possible. The same time he was able to close the eye as soon as he willing doing that. The measurements continued until the next eye closing. 


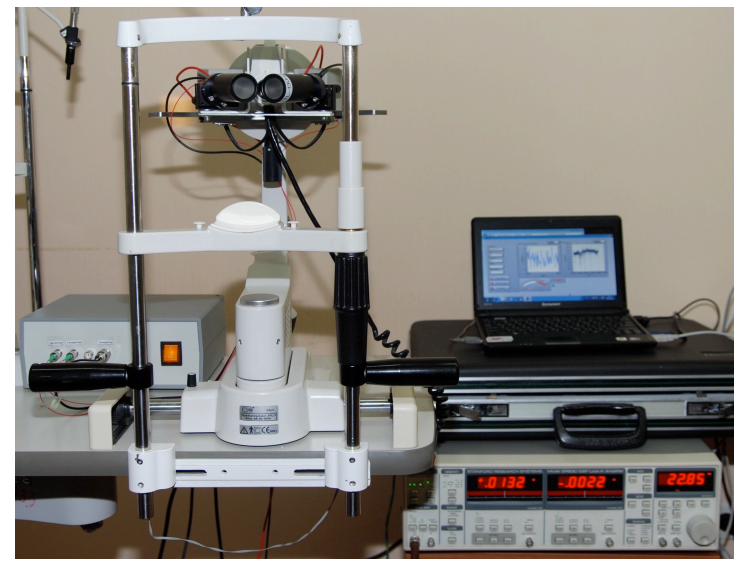

Fig. 1. THz reflectometer setup embedded into ophthalmic apparatus

The $\mathrm{THz}$ reflectivity of the cornea demonstrates the dehydration dynamics (Fig.2). Such dependency may be fitted by the special function and decreases with the time. At a time of eye closing the reflectivity decreases and remains constant while the eye is closed. After the next eye opening the reflectivity restores to the value similar one of the previous cycle. Based on the results of preliminary studies, it can be possible to measure of pre-corneal tear film dynamics.

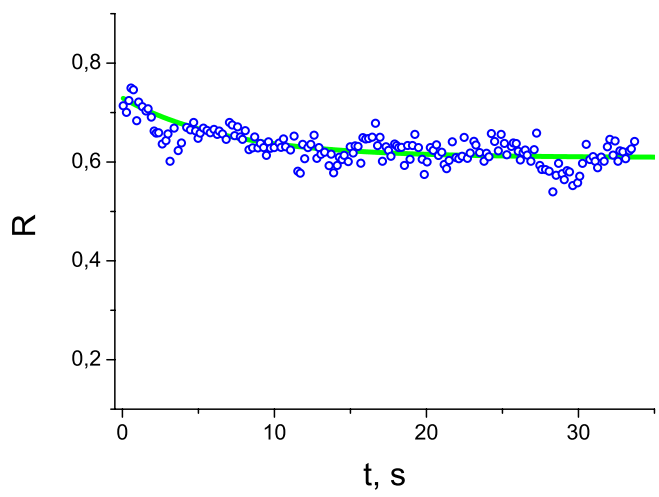

Fig. 2. Reflection coefficient of the ocular surface system as a function of time

Fig. 2 illustrates an example of the ocular surface system $\mathrm{THz}$ reflection coefficient versus time. This graph was obtained for the cornea of testee human without any claims. In case of complains on dryness in the eyes and suspicion of dry eye syndrome the dynamics of reflection coefficient change would be more changeable. Norn tests for each testee human were performed and the behavior of the reflection coefficient dynamics for these patients possesses good correlation with the Norn test results.

The value of $\mathrm{THz}$ reflection coefficient at the right edge of the graph in Fig. 2 corresponds to the case of critical thinning of the tear film and very close to the value of tear film break up case. This value could be taken for estimation the reflection coefficient of the corneal tissue and calculation of the hydration level of this tissue.

To confirm the detected correlations, similar studies were carried out for three groups of subjects. In the first group of 10 people, the eyes of people who did not express subjective complaints of discomfort in the eyes or excessive lacrimation were considered.

The second group represented a sample of people with instability of tear film established by Norn test and subjective complaints of dryness. The third group included eyes with hypersecretion of tear glands confirmed by Shirmer test. These groups of tested humans with eye abnormalities included 13 and 7 subjects respectively.

The results of these studies, presented in the form of a histogram in Fig. 3, show a correlation between the value of the linear function slope and the established diagnosis.

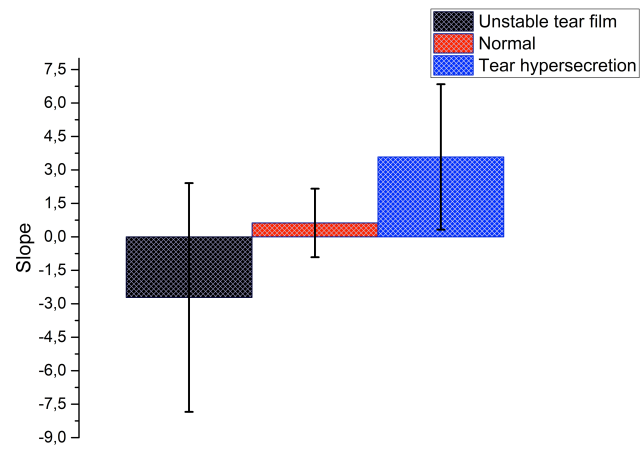

Fig. 3. Histogram of function slope coefficient for different eye type diseases

We showed the results of applications of the $\mathrm{THz}$ reflectometry to sensing of the ocular surface system and pre-corneal tear film. Our study allows the dynamic control of pre-corneal tear film thinning and sensing of corneal tissue hydration level in clinical applications in vivo.

This work was supported by RFBR (projects No.15-29-03900 and 17-00-00270).

\section{References}

1. K.M.Meek. The Cornea and Sclera. Boston, MA:Springer, 2008.

2. V.V.Brzheskij, G.B.Egorova and E.A.Egorov. Syndrome of 'dry eyes' and diseases of the eye surface: clinic, diagnosis and treatment. Moscow: GEOTAR-Media, 2016.

3. Z.D.Taylor, J.Garritano, S.Sung, et al. THz and $\mathrm{mm}-$ Wave Sensing of Corneal Tissue Water Content: In Vivo Sensing and Imaging Results. IEEE Transactions on Terahertz Science and Technology, vol.5, pp.184-196, 2015.

4. I.Ozheredov, M.Prokopchuk, M.Mischenko, et al. In vivo $\mathrm{THz}$ Sensing of Eye Cornea. Laser Physics Letters, vol.15, 055601, 2018 